\title{
Development of a Novel Web-Based Tool to Improve Emergency Depart- ment Communication with General Practitioners: A Needs Assessment Survey
} \author{
$\operatorname{ard}^{1,2}$ and Borgundvaag Bjug ${ }^{1,2, *}$ \\ ${ }^{I}$ Department of Family and Community Medicine, University of Toronto, Toronto, Canada \\ ${ }^{2}$ Schwartz-Reisman Emergency Centre, Mount Sinai Hospital, Toronto, Canada \\ ${ }^{3}$ The Granovsky-Gluskin FamilyMedicine Centre, Mount Sinai Hospital, Toronto, Canada
}

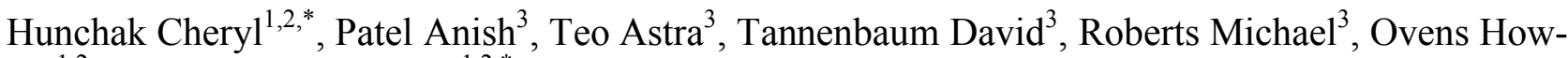

\begin{abstract}
Background: Communication of emergency department (ED) visit information to general practitioners (GP) is often inadequate and can negatively impact on patient care. Further, the use of email as a communication tool between GP and ED providers has been not been well explored.

Objectives: We sought to assess the desirability, feasibility and ideal functionalities of a novel web-based, automated postED visit communication tool for GPs.

Methods: A cross-sectional needs assessment survey was conducted among the top 300 referring GPs to a single ED in Toronto, Canada. The main outcome measures were: current GP awareness of patient ED visits and anticipated uptake of an electronic notification and health record communication tool.

Results: One hundred ninety-eight physicians responded (66\% response rate). Fifty-eight percent of GPs were unaware or only sometimes aware of patients' ED visits. Nearly all (94\%) would welcome an automated electronic system to communicate post-ED discharge health information in real-time. Two-thirds $(67 \%)$ were in favour of their patients having online access to their own health records. Physicians less than 50 years of age were more likely than those greater than 50 to use both an office computer with internet and email access (96\% versus 69\%)and an electronic medical record (EMR;57\% versus $41 \%)$.

Conclusions: This needs assessment survey highlights an unmet need for improved ED-GP health record communication and suggests that GP uptake of a novel web-based post-ED visit notification and health record transfer system would be high.
\end{abstract}

Keywords: Communication tool, Emergency care information systems, Emergency department, Medical informatics application, Patient discharge, Primary care physicians, Web-based technology.

\section{INTRODUCTION}

Reliable communication of health record information between the emergency department (ED) and community general practitioners (GPs) is a cornerstone of quality patient care. In reality, however, ensuring that GPs have timely access to relevant clinical results following patients' ED visits is challenging. This is for myriad reasons, including illegibility of handwritten records, incomplete delivery of printed records by patients, incomplete information available at the time of patient discharge, and incomplete contact information for community physicians.

Studies have shown that GPs are often unaware when their patients are seen in the ED [1-3]. Results from ED

*Address correspondence to these authors at the Mount Sinai Hospital, 600 University Avenue, Room 206, Toronto, Ontario, M5G 1X5; Canada. Tel: 416586 5058; Fax: 416586 4719; E-mail: chunchak@gmail.com visits are inconsistently or infrequently communicated to GPs in a timely manner, often with more than two weeks delay before reports are received [2]. A survey of ED chiefs by Stiell et al. [1] found that ED communication with GPs most often consisted of the handwritten ED treatment record being sent by mail and that many doctors felt the quality of communication was inadequate. Further, poor communication between the ED and community physicians has been shown to increase overall resource utilization and contribute to unnecessary duplication of tests and specialist referrals [4].

General practitioners, ED physicians, and indeed patients, believe that communication gaps between acute care and community physicians negatively impact on continuity of care $[1,3]$. Studies have suggested that a standardized electronic ED discharge system could improve current communication $[5,6]$. One Canadian study demonstrated that introduction of an electronic standardized communication system significantly improved continuity of patient care fol- 


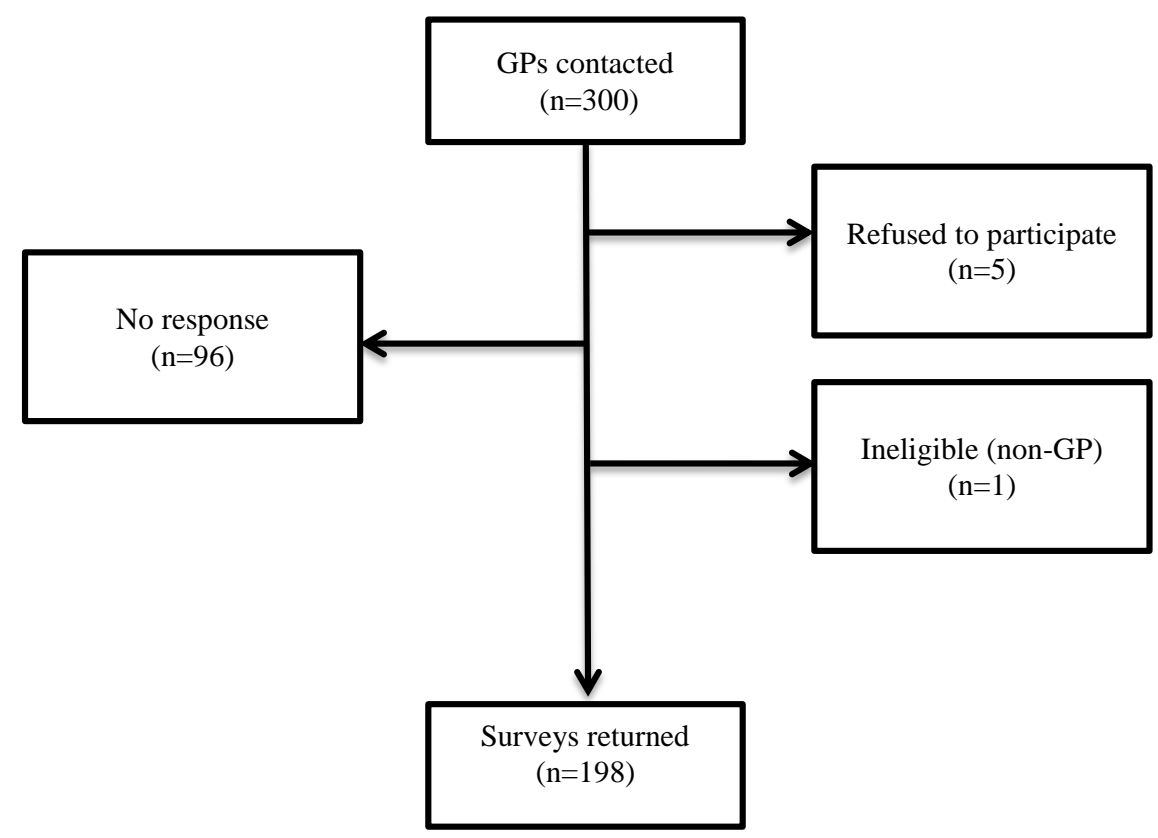

Fig. (1). Flowchart demonstrating survey response rate.

lowing ED discharge, specifically contributing to a higher rate of information transfer and improved GP knowledge of patient $E D$ visits [6].

To date, we are aware of only one hospital in the Greater Toronto Area, Canada, using an electronic notification system to convey ED visit information to GPs [7]. To determine the desirability, feasibility and functionality of an automated web-based notification system to alert GPs when their patients had been seen in the ED, we conducted a needs assessment survey among GPs who frequently refer patients to a single hospital in Toronto, Canada.

\section{METHODS}

\section{Selection of Survey Subjects}

The needs assessment consisted of a written survey administered to 300 GPs in the Greater Toronto Area who, between the period of June 2009 and July 2010, were identified by a single hospital's records as the top referring community GPs to the ED or who were most frequently identified by ED patients as their GP. The College of Physicians and Surgeons of Ontario (CPSO) directory was used to confirm the practice status and contact information of identified physicians. Physicians were excluded if they were not in general practice.

\section{Survey Methodology}

The survey instrument comprised 16 questions regarding current internet access, desired web portal functionalities and methods of communication between the hospital ED and GPs (Appendix 1). During a one month period in January 2011, surveys were mailed out to physicians' offices in accordance with the mail survey research methodology previously described by Dillman [8]. Physicians were given the option of returning the survey by mail or fax. Those physicians who had not responded after 30 days were contacted and offered a chance to complete the survey by phone according to a standardized telephone interview script. The survey was anonymous insofar as the investigators were blinded to the responders' information; only the research assistant had access to the codes linking individual surveys to GPs in order to identify non-responders.

Respondents were asked to report their age, gender, number of years practicing medicine in Canada and number of practice locations. With respect to the current state of EDGP communication, GPs were asked how often they were aware that their patients had been seen in the hospital ED and how often they were currently receiving any form of post-ED visit communication including unsolicited faxes, hand delivered copies of reports, and requests for hospital medical records. Physicians were questioned about the desired types of information that should be provided, the duration of time that online health information should be made available, and the desirability of patients accessing their own health information through the web portal.

\section{Statistical Analysis}

Descriptive statistical analysis was completed using SPSS for Windows. Response frequencies were calculated for each survey item.

This study was approved by the Mount Sinai Hospital Research Ethics Board in Toronto, Canada. The results of a subsequent pilot study of the design and implementation of the web portal notification system informed by this needs assessment are pending separate publication.

\section{RESULTS}

\section{Study Population}

The survey achieved a $66 \%$ response rate, with $198 / 300$ surveys completed (Fig. 1). Table 1 highlights the demographic characteristics of the surveyed GPs. The majority of 
Table 1. Baseline Characteristics of Surveyed GPs

\begin{tabular}{|c|c|c|}
\hline Gender (\%) & Male & 64 \\
\hline \multirow{4}{*}{ Age (in years; \%) } & $\leq 39$ & 9 \\
\hline & $40-49$ & 19 \\
\hline & $50-59$ & 31 \\
\hline & $\geq 60$ & 40 \\
\hline \# Years in practice (mean) & \multicolumn{2}{|c|}{27} \\
\hline \# Practice locations (mean) & \multicolumn{2}{|c|}{1.4} \\
\hline
\end{tabular}

Table 2. Current GP Practices Relating to Internet Access and Use (\%)

\begin{tabular}{|c|c|}
\hline \multicolumn{1}{|c|}{ Access to Computer with Internet/Email in Office } & \multicolumn{2}{|c|}{77} \\
\hline \hline Use of EMR in office & 46 \\
\hline Frequency of awareness that a patient was seen in the ED ‘often' or 'always' & \multicolumn{2}{|c|}{ Unsolicited fax } \\
\hline \multirow{2}{*}{ Frequency of received communication from ED ‘often' or 'always' } & Patient hand delivers report \\
\cline { 2 - 3 } & \multicolumn{2}{|c|}{ GP requests from hospital records } \\
\hline
\end{tabular}

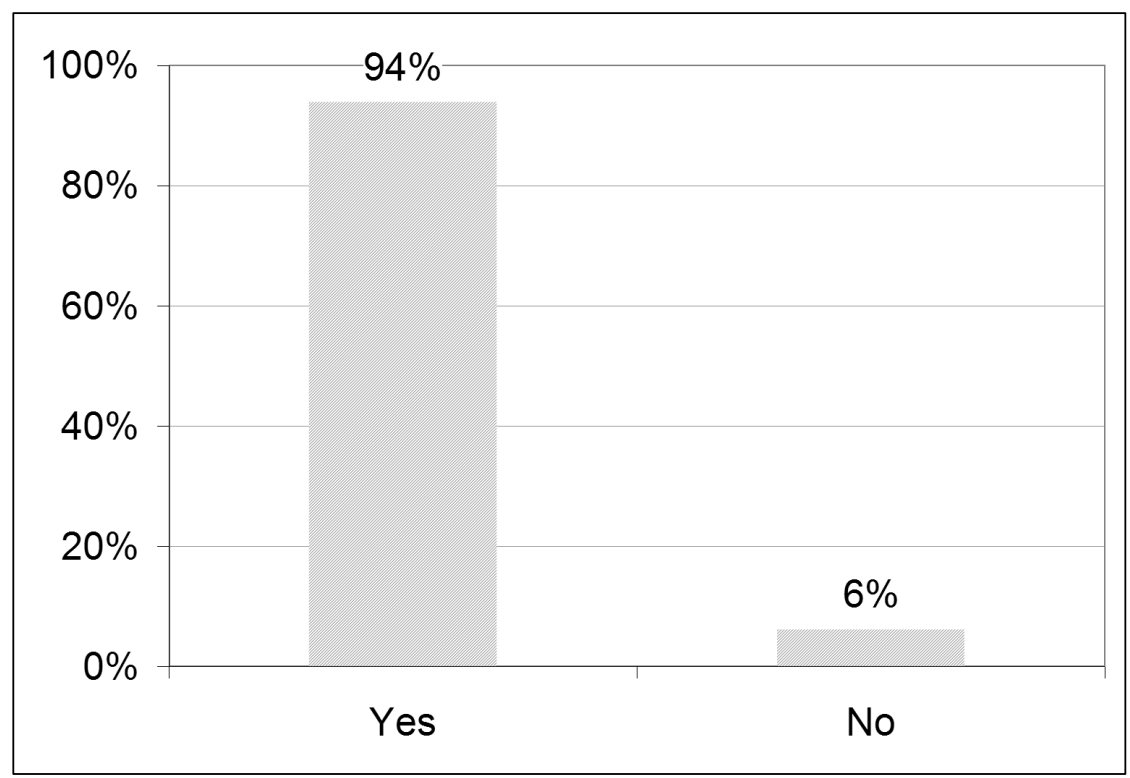

Fig. (2). Interest in a novel web-based post-EDvisit electronic communication system.

respondents were male $(64 \%)$ and were aged 50 years or older $(71 \%)$. The average number of years in practice was 27. Respondents maintained an average of 1.4 practice locations.

\section{Outcomes}

Overall, the majority (59\%) of physicians felt they were either 'never' or only 'sometimes' aware that their patients had been seen at the hospital ED and78\%'never' or only 'sometimes' received ED discharge information by fax. Paper-based forms of communication were poor, with only
$41 \%$ 'often' or 'always' receiving hand-delivered copies of ED reports from patients, which is the current standard method of ED-GP communication (Table 2). Nearly all (94\%) were interested in using an electronic communication system to improve information exchange with the hospital ED (Fig. 2).

Most respondents (76\%) thought they would use such a system 'regularly' or 'always' (Table 3). The difference in anticipated frequency of usage between physicians younger and older than 50 years of age was small (71\% versus $78 \%$ respectively). A greater number of GPs preferred that visit 
Table 3. Expressed Preferences for Web Portal Design (\%)

\begin{tabular}{|c|c|c|}
\hline Preference for Access to Web-Based Post-ED Visit Notification System & Yes & 94 \\
\hline \multirow{3}{*}{ Anticipated usage of web-based notification system } & Infrequently & 3 \\
\hline & Occasionally & 21 \\
\hline & Regularly & 54 \\
\hline \multirow{2}{*}{ Preferred email address for notifications to be sent to } & Personal & 30 \\
\hline & Office & 68 \\
\hline \multirow[b]{2}{*}{ Preferred method of notification of ED visit } & Fax & 57 \\
\hline & Email & 40 \\
\hline \multirow{2}{*}{ Preferred content of access to diagnostic imaging results } & Report only & 80 \\
\hline & Images and report & 20 \\
\hline \multirow{5}{*}{ Preferred length of time for access to on-line results } & 2 weeks & 12 \\
\hline & 4 weeks & 29 \\
\hline & 6 weeks & 6 \\
\hline & 8 weeks & 9 \\
\hline & As long as possible & 45 \\
\hline
\end{tabular}

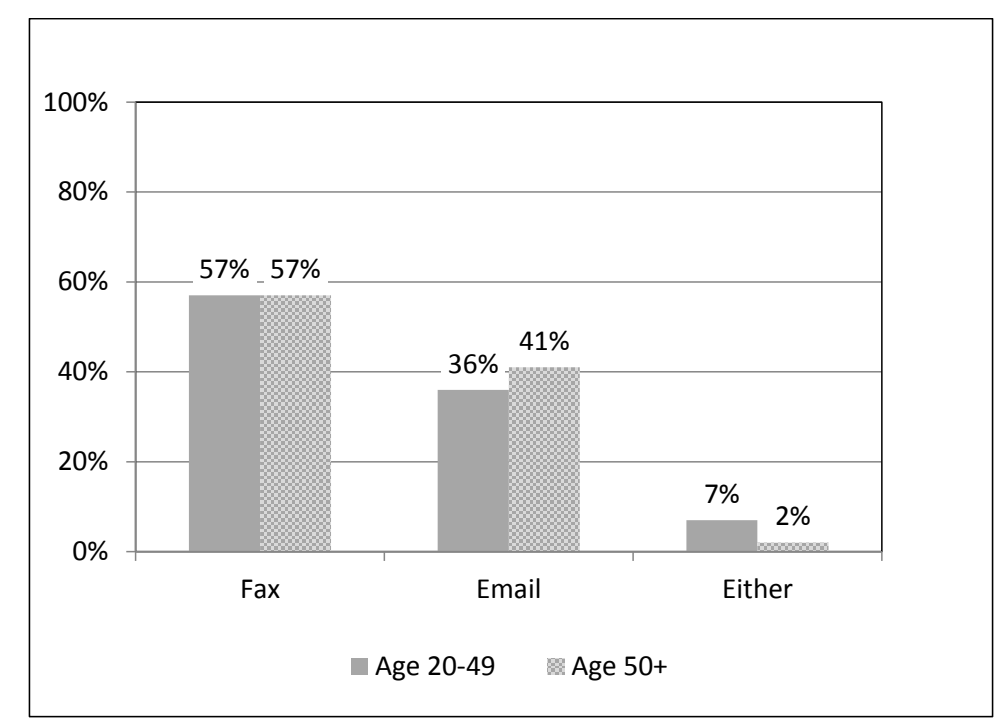

Fig. (3). Preferred method of post-ED visit notification by age.

notification be communicated via fax $(57 \%)$ rather than by email (40\%) (Fig. 3). The preference for faxed communications was similar between those younger and older than 50 years of age ( $57 \%$ versus $56 \%)$.
Respondents indicated a preference for electronic information to be sent to an office email account (68\%) rather thana personal email account (30\%). Most physicians (86\%) preferred that all available reports and test results be pro- 


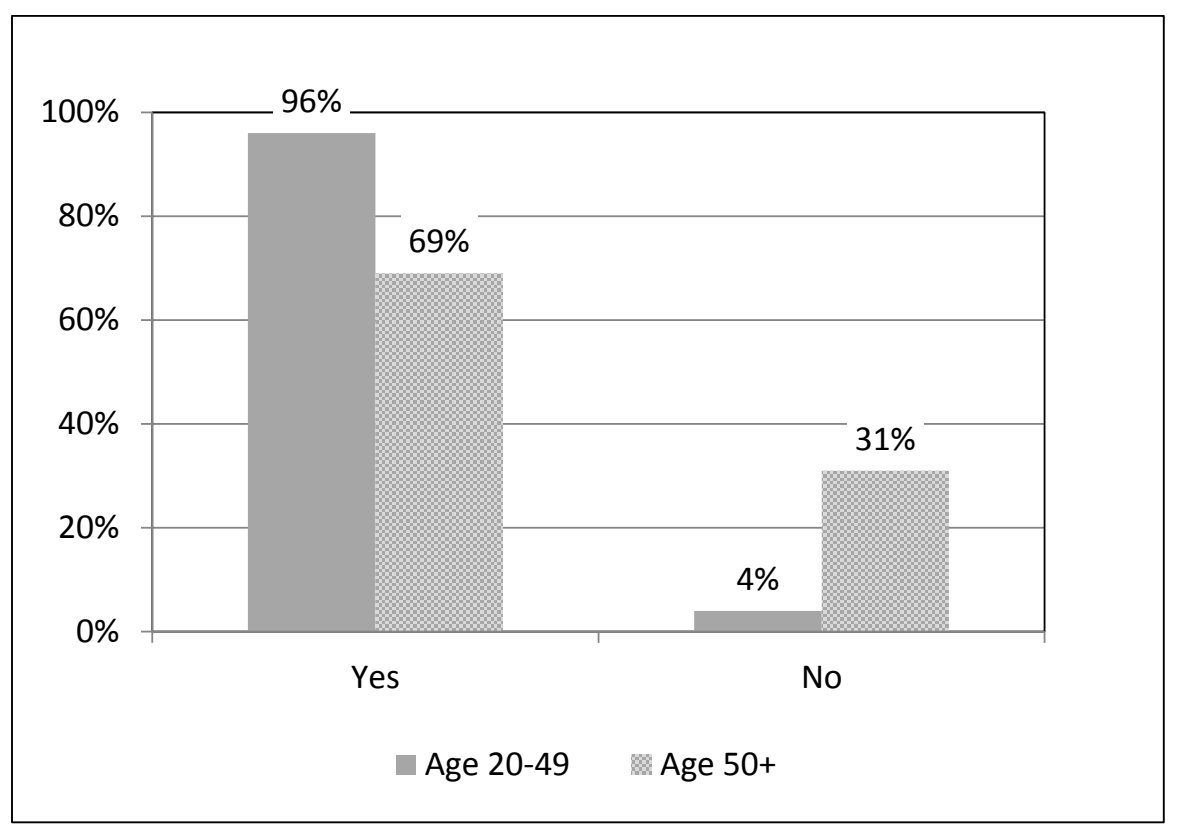

Fig. (4). Availability of an office computer with internet and email access by age.



Fig. (5). Use of electronic medical records (EMRs) among GPs by age.

vided. More than three quarters of GPs (77\%) had internet and email access in their office. Physicians less than 50 years of age were more likely to have a computer with internet and email access in their office compared to those greater than 50 years of age ( $96 \%$ versus $68 \%$ respectively; Fig. 4 ).

Nearly half (46\%) of GPs were using an electronic medical record (EMR) system. A greater number of younger physicians were using EMR compared to older physicians ( $57 \%$ versus $41 \%$ respectively; Fig. 5).

\section{DISCUSSION}

This study demonstrates a clear unmet need among GPs for improved timely communication of patient health information following ED visits. This is consistent with previous study findings $[1,3]$. The desire to embrace web-based communication platforms is clear. Timely electronic communication of post-ED visit health records to GPs has the potential to achieve the following: 1) reduce unnecessary duplication of tests and referrals, 2) improve patient continuity of care, 3) improve patient and family perception of careand 4) enhance 'circle of care' relationships between hospital and community physicians.

There are several limitations to consider in this study. It is difficult for GPs to know, at baseline, how often they are currently unaware of when their patients have been seen in the ED. If patients do not hand-deliver the written ED assessment note, or do not follow up with their GP about the chief complaint for which they were seen in the ED, the true 
total number of ED visits incurred by GP patients remains uncertain. Due to the self-limited nature of many conditions for which patients seek ED care, the true denominator of GP patients seen in the ED is likely to be larger than reported, and suggests that GP awareness of ED visits may be even lower than realized. Second, the survey response rate of $66 \%$ was lower than the desired target of $70 \%$. An email-based survey was considered in the study design phase, and may have increased our response rate due to ease of survey return. However, this method risked biasing the results in favour of the web portal on account of reaching GPs already savvy with email and internet. In retrospect, based on the older age demographic and communication preferences favouring faxed ED visit notifications in our results, an email-based survey would have been unlikely to increase our survey response rate. Third, the demographic characteristics of the top referring GPs to the study hospital ED may not reflect the general cadre of GPs in the Greater Toronto Area and likely understates the perceived need for a web-based ED notification system to improve ED-GP communication. Finally, it is worth noting that the 'physician' with the highest number of referrals during the study period was listed as 'No General Practitioner'. This highlights an important potential future application whereby patients could choose to access their own ED visit results online and share them with subsequent health providers, improving continuity of care for patients who lack a consistent primary care provider.

The expressed preference for fax versus email notification of ED visits was unanticipated, especially among younger GPs. Based on written survey comments, we hypothesized the following possible reasons for this sentiment: 1) relative lack of internet and email access in the office, 2) concern about maintaining patient confidentiality in email communications, 3) inability to integrate information obtained from the web portal system with pre-existing EMR systems and 4) preference for maintaining the responsibility of calling patients to follow up on ED visits with office staff via received faxes, which is currently common standard practice. This expressed preference for fax communication pertained only to receipt of the automated post-ED visit notification message; the patient health information provided to GPs would still then be accessed online. An overwhelming majority of surveyed GPs (94\%) were in favour of this, as previously outlined.

As anticipated, there was a significant difference in current internet and EMR usage and accessibility between age groups (57\% among GPs $<$ age 50 versus $41 \%$ among GPs $\geq$ age 50). Current trends suggest, however, that over time, increased familiarity and uptake of EMR systems and emailbased patient care communications among GPs as a group is inevitable [7].
The need for a standardized web-based ED-GP visit notification system in hospitals is clear. Implementation of such a system has far-reaching implications. Automated electronic health record communication tools currently represent an underutilized resource to link hospital and community physicians in near real-time. Web portal technology has the capacity to extend the traditional 'hospital' network to include community physicians in the same way that EMR systems have improved inter-specialist access to patient health records within hospitals. Applications of the health information web portal concept extend beyond the ED and may have greatest relevance and benefit for patients requiring complex medical care. Web-based patient visit notification systems take the 'circle of care' concept one step further than traditional EMR systems by proactively notifying shared-care providers of patient visits to alert them to changes in the patient's medical status, rather than simply providing a passive repository of patient records. This novel 'provider-alert' functionality has broad applications for general and specialist physicians caring for patients with common medical conditions requiring coordinated multi-disciplinary care including diabetes, a trial fibrillation and inflammatory bowel disease, to name a few.

As novel web-based health record access systems develop, many questions remain. Privacy implications are considerable and an assessment of patient demand for online access to their own portable health records is also needed. Pilot studies ought to be conducted to assess the utility and effectiveness of these novel communication tools.

\section{CONCLUSION}

The use of email as a tool to enable real-time communication between ED and community physicians remains unrealized $[9,10]$.Our results suggest that there is an important unmet need for the development and implementation of webbased platforms to communicate post-ED visit notification and health record information to community GPs.

\section{CONFLICT OF INTEREST}

The authors confirm that this article content has no conflicts of interest.

\section{ACKNOWLEDGEMENTS}

This study was funded by the Mount Sinai HospitalUniversity Health Network Academic Medical Organization Innovation Fund in Toronto, Canada. The authors would like to thank Sally Carver for here assistance with manuscript preparation

\section{APPENDIX 1. NEEDS ASSESSMENT SURVEY}

1. Do you have access to a computer with e-mail and internet in your office?

$$
\text { Yes }
$$

$$
\text { No }
$$

2. How many locations do you currently practice medicine at?

3. How many years have you been practicing medicine in Canada?

4. What is your age range (in years)? 

20-29
30-39
40-49
$50-59$
60-69
$70+$

5. Please indicate your gender:

Male

Female

6. Would you like to have access to a web-based system that will notify you when your patients are seen in the emergency department?

Yes No

If you answered Yes to question 6, please proceed to question 7.

If you answered No to question 6, please take a moment to tell us why you would not be interested in such a system. This is the end of the questionnaire, thank you for participating.

7. From the list below, please indicate how often you think you would use such a system:
A. Infrequently
B. Occasionally
C. Regularly
D. Always

8. Would you prefer that notifications be sent to your personal email account or to an office email account?

\section{Personal Office}

9. Please select the statement below that most accurately reflects the type of information you would like to receive regarding your patient's visits to the emergency department:

A. I would like to have access to all reports and tests which are part of my patient's medical record from their ED visit

B. I would prefer to simply be notified that my patient was seen in the emergency department along with the discharge diagnosis

10. With regards to diagnostic imaging (X-Ray, CT, Ultrasound, MRI), please circle one of the following statements which most accurately reflects your views:

A. I would like to have access to imaging reports only

B. I would like to have access to imaging reports and the actual images

11. Would you prefer to receive notification that your patient has been seen in the emergency department by fax or by email?

$$
\text { Fax Email }
$$

12. Given that electronic information regarding your patient's visit cannot be made available indefinitely, how long do you think it should be available?
A. 2 weeks
B. 4 weeks
C. 6 weeks
D. 8 weeks
E. As long as possible

13. At the present time, how often are you aware that one of your patients was seen in the Mount Sinai Hospital emergency department?
A. $\quad$ Never (please proceed to question 15)
B. Sometimes
C. Often
D. Always

14. If you are aware that your patient has been seen in the emergency department, how often do you currently receive any of the following types of communication:
A. Unsolicited fax
Never Sometimes Often Always
B. Patient hand delivers copies of reports
Never Sometimes Often Always 


\section{I have to request it from medical records Never Sometimes Often Always}

\section{Do you use an EMR in your office?}

Yes

No

16. Are you in favour of your patients being able to access electronic copies of their lab results, microbiology reports and imaging studies performed during their emergency department visit?

Yes No

17. If you are interested in receiving your patients' post-discharge ED results via the web-based system described above, please provide us with the current email address you use for work-related communication and your preferred office contact information below. The email address you provide will not be shared with any other parties and will be used exclusively for the purposes of the secure web-based portal system. We plan to conduct a future pilot study and when the system is ready for full operation we would be delighted to include you in this initiative.

\section{REFERENCES}

Stiell AP, Forster AJ, Stiell IG, Walraven C. Maintaining continuity of care: a look at the quality of communication between Ontario emergency departments and community physicians. CJEM 2005; 7(3): 155-61.

[2] Sherry M, Edmunds S, Touquet R. The reliability of patients in delivering their letter from the hospital accident and emergency department to their general practitioner. Arch Emerg Med 1985; 2(3): 161-4.

[3] Stiell A, Forster AJ, Stiell IG, et al. Prevalence of information gaps in the emergency department and the effect on patient outcomes. CMAJ 2003; 169: 1023-8.

[4] Lang E, Afilalo M, Vandal AC, et al. Impact of an electronic link between the emergency department and family physicians: a randomized controlled trial. CMAJ 2006; 174(3): 313-8.
[5] Wass AR, Illingworth RN. What information do general practitioners want about accident and emergency patients? J Accid Emerg Med 1996; 13: 406-8.

[6] Afilalo M, Lang E, Leger R, et al. Impact of a standardized communication system oncontinuity of care between family physicians and the emergency department. CJEM 2007; 9(2): 79-86.

[7] Yau GL, Williams AS, Brown JB. Family physicians' perspectives on personal health records. Can Fam Physician 2011; 57: e178-84.

[8] Dillman DA. Mail and Internet Surveys. $2^{\text {nd }}$ ed. Toronto: John Wiley \& Sons, Inc 2000

[9] Moyer CA, Stern DT, Dobias KS, Cox DT, Katz SJ. Bridging the electronic divide: patient and provider perspectives on email communication in primary care. Am J Manag Care 2002; 8(5): 427-33.

[10] Gaster B, Knight CL, DeWitt DE, Sheffield JVL, Assefi NP, Buchwald D. Physicians' use of and attitudes toward electronic mail for patient communication. J Gen Intern Med 2003; 18(5): $385-9$. 